\title{
ANÁLISE DAS NOTIFICAÇÕES DE ACIDENTES PEDIÁTRICOS NA CIDADE DE SÃO PAULO
}

\author{
NOTIFICATIONS OF PEDIATRIC ACCIDENTS IN SÃO PAULO, SP, BRAZIL
}

\author{
Cintia Leci Rodrigues ${ }^{\mathrm{a}^{*}}$, Jane de Eston Armond ${ }^{\mathrm{b}}$, Ana Paula Marconi lamarino ${ }^{\mathrm{c}^{*}}$, \\ Ana Carolina Giolo dos Santos ${ }^{\mathrm{d}^{*}}$, Rodrigo de Eston Armond ${ }^{\mathrm{e}}$, Carlos Górios ${ }^{\mathrm{f*}}$ \\ akikarodrigues@hotmail.com, bjearmond@terra.com.br, canapaulamarconi@gmail.com, danacarolinagiolo@hotmail.com, \\ erodrigo@wbcsaude.com.br, 'gorios@terra.com.br \\ *Universidade de Santo Amaro - São Paulo (SP), Brasil \\ **Centro Universitário São Camilo - São Paulo (SP), Brasil
}

Data de recebimento do artigo: 17/09/2016 Data de aceite do artigo: 15/02/2017

\section{RESUMO}

Objetivo: Analisar os dados de acidentes com crianças registrados na rede de atendimento do Sistema Único de Saúde (SUS) e no Sistema de Vigilância de Violências e Acidentes (Sivva) do município de São Paulo (SP) no período de janeiro a dezembro de 2015. Métodos: Trata-se de um estudo transversal de abordagem quantitativa, descritivo, retrospectivo. O estudo abrangeu a totalidade de 16.847 crianças com idade entre 0 a 9 anos, vítimas de acidentes, notificadas no Sistema de Informação para a Vigilância de Violências e Acidentes da Secretaria Municipal de Saúde da cidade de São Paulo, onde são registradas as notificaçóes de acidentes e violência por meio da ficha de notificação de casos suspeitos ou confirmados. Resultados: Em crianças de todas as idades da faixa pesquisada e em ambos os sexos, as quedas acidentais foram o tipo de acidente mais registrado. Os acidentes ocorreram em crianças com faixa etária entre 0 e 4 anos $(63,9 \%)$ e 5 e 9 anos (36,1\%). Em relação ao sexo, 39,1\% dos acidentes ocorreram no sexo feminino e $60,9 \%$ entre crianças do sexo masculino. Conclusáo: Este estudo mostrou que o principal tipo de acidente entre crianças, a queda doméstica, ocorre predominantemente no ambiente doméstico e com crianças do sexo masculino. Notou-se com esta pesquisa que estamos diante de crianças vulneráveis, que necessitam de cuidados específicos, principalmente preventivos, em açôes intersetoriais com órgãos e profissionais da saúde e educação envolvidos no acompanhamento do crescimento e desenvolvimento das crianças.

Palavras-chave: Acidentes; criança; prevenção e controle.

\section{ABSTRACT}

Objective: To analyze data of accidents with children recorded in the service network of the Unified Health System (SUS) and in the Surveillance System for Violence and Accident (VIVA) of São Paulo (SP) in the period of January to December 2015. Methods: This is a cross-sectional study, with quantitative, descriptive and retrospective approach. The study covered the totality of 16,847 children aged 0 to 9 years, victims of accidents, reported in VIVA of the Municipal Health Secretariat of São Paulo, in which accidents and violence reports are recorded by the suspected or confirmed case notification form. Results: In children of all ages of the surveyed group and in both genders, accidental falls were the most recorded type of accident: they occurred in children aged between 0 and 4 years (63.9\%); 5 to 9 years (36.1\%). Regarding gender, $39.1 \%$ of the accidents occurred in females and $60.9 \%$ among male children. Conclusion: This study showed that the main type of accident were accidental falls occurred predominantly at home and with males. It was noted in this study that we are facing vulnerable children who need special care, mainly preventive, in intersectoral action with health and education agencies and professionals that are involved in monitoring the growth and development of children.

Keywords: Accidents; child; prevention and control. 


\section{Introdução}

Os acidentes e violências compóem as chamadas causas externas, que representam uma das principais causas de mortalidade entre crianças e adolescentes, tornando-se cada vez mais um relevante problema em saúde pública ${ }^{1,2}$. Por isso, são importantes os estudos acerca do tema, que ajudam a compreender a distribuição, as causas, as características, a magnitude e os aspectos relacionados à ocorrência desses acidentes ${ }^{1}$.

Os acidentes também são responsáveis por eventos não fatais e sequelas que podem afetar negativamente a qualidade de vida de crianças e adolescentes ${ }^{2}$. Segundo Xavier-Gomes et al. ${ }^{3}$, o grupo infantil é o mais vulnerável: quanto mais jovem e imatura for a criança, menor será sua percepção de risco, e maior sua exposição aos acidentes. A vulnerabilidade da criança é variável em função do nível de coordenação de seu sistema nervoso, aptidão motora, senso de percepção de risco e outros fatores ${ }^{3}$.

As lesóes, os traumas e as mortes decorrentes de acidentes na infância geram custos aos serviços de saúde e à sociedade que se traduzem em demanda aos serviços sociais, internaçóes hospitalares, reabilitação, danos mentais e emocionais para as vítimas e familiares, além da perda de anos potenciais de vida nos casos fatais ${ }^{4}$.

O estabelecimento de políticas públicas para a prevenção e o controle dos acidentes deve ter por base um quadro o mais completo possível do problema. Por isso, é necessário conhecer também as lesóes de menor gravidade, que não determinam mortes ou internaçoóes, mas que são responsáveis por uma grande demanda nas emergências ${ }^{5}$.

Este artigo tem como objetivo analisar os dados de acidentes com crianças registrados na rede de atendimento do Sistema Único de Saúde (SUS) e no Sistema de Vigilância de Violências e Acidentes (Sivva) do município de São Paulo (SP) no período de janeiro a dezembro de 2015.

\section{Métodos}

Trata-se de um estudo transversal de abordagem quantitativa, descritivo, retrospectivo, que abrangeu a totalidade de 16.847 crianças com idade entre 0 e 9 anos, vítimas de acidentes notificados no Sistema de Informação para a Vigilância de Violências e Acidentes (Sivva) da
Secretaria Municipal de Saúde da cidade de São Paulo, onde são registradas as ocorrências de acidentes e violência por meio da ficha de notificação de casos suspeitos ou confirmados ${ }^{6}$. Foram considerados os registros realizados durante o período de janeiro a dezembro de 2015.

As variáveis utilizadas para o estudo foram: idade, sexo, tipo de acidente (queda, afogamento, arma branca, choque elétrico, fogo/incêndio, intoxicação/envenenamento, mordedura, outras queimaduras e sufocação), agente da intoxicação, diagnóstico de lesão, evolução do caso (encaminhamento para serviços, alta hospitalar, internação, óbito no atendimento ou recebido em óbito), local do acidente (residência, via pública, creche, escola), tipo de deficiência (física, mental, visual) e período da ocorrência do acidente (dia ou noite).

Todas as variáveis basearam-se nos dados de domínio público informados pelo Sivva.

O Sivva tem como fonte a notificação de atendimentos de vítimas de violências e acidentes por profissionais de saúde. Essa notificação se faz por meio de um instrumento específico (ficha de notificação de casos suspeitos ou confirmados de violência e acidentes). Dessa forma, o banco de dados do Sivva não é representativo da totalidade dos acidentes ou violências, mas dos casos que incidem nos serviços de saúde.

As variáveis de interesse foram coletadas no banco de dados e tabuladas. Em seguida, foram realizadas análises descritivas para a caracterização da amostra por meio de cálculo de frequências absolutas e relativas.

$\mathrm{O}$ projeto de pesquisa dispensou aprovação do Comitê de Ética em Pesquisa da Universidade, pois trabalhou com dados secundários de banco de dados de domínio público, conforme preconizado pela Resolução CNS no 466/12.

\section{Resultados}

Durante o período estudado, foram registrados 16.847 acidentes envolvendo crianças na cidade de São Paulo. Os acidentes ocorreram em crianças com faixa etária entre 0 e 4 anos $(63,9 \%)$ e 5 e 9 anos $(36,1 \%)$.

Em relação ao sexo, 39,1\% dos acidentes ocorreram com crianças do sexo feminino e $60,9 \%$ com crianças do sexo masculino.

Tabela 1: Tipo de acidentes ocorridos na infância, segundo sexo, na cidade de São Paulo, 2015.

\begin{tabular}{lcccc} 
Tipo de acidente & Feminino & $\mathbf{f ( \% )}$ & Masculino & $\mathbf{f ( \% )}$ \\
Queda & 5338 & 81,1 & 8137 & 79,3 \\
Afogamento & 1 & 0,0 & 3 & 0,0 \\
Arma Branca & 11 & 0,2 & 24 & 0,2 \\
Choque elétrico & 0 & 0,0 & 4 & 0,0 \\
Fogo/Incêndio & 3 & 0,0 & 2 & 0,0 \\
Intoxicação/envenenamento & 40 & 0,6 & 58 & 0,6 \\
\hline
\end{tabular}


Tabela 1: Continuação.

$\begin{array}{lcccc}\text { Tipo de acidente } & \text { Feminino } & \mathbf{f ~ ( \% )} & \text { Masculino } & \mathbf{f}(\mathbf{\% )}) \\ \text { Mordedura } & 3 & 0,0 & 7 & 0,1 \\ \text { Outras queimaduras } & 246 & 3,7 & 300 & 2,9 \\ \text { Sufocação } & 9 & 0,1 & 10 & 0,1 \\ \text { Ignorado } & 930 & 14,1 & 1719 & 16,7 \\ \text { Total } & 6582 & 100,0 & 10265 & 100,0\end{array}$

O principal tipo de acidente na infância foram as quedas acidentais em ambos os sexos e em todas as idades da faixa pesquisada. Vale ressaltar que os acidentes de intoxicação/envenenamento e outras queimaduras foram maiores em crianças com idade entre 0 e 4 anos de idade.

Sobre os agentes de intoxicaçáo/envenenamento, os principais notificados foram: produto químico industrial $(4,1 \%)$, saneantes $(3,1 \%)$, ácido acetilsalicílico $(3,1 \%)$, agrotóxico de uso domiciliar $(3,1 \%)$, chumbinho $(2,0 \%)$ e drogas de abuso $(1,0 \%)$.

Os diagnósticos de lesão (CID-10) mais registrados foram: traumatismo superficial múltiplo do pescoço $(15,6 \%)$, ferimento da cabeça $(10,4 \%)$, traumatismo superficial da cabeça, parte não especificada $(10,1 \%)$.

Em relação à evolução do caso, das crianças que sofreram acidentes no período analisado $79,7 \%$ tiveram alta hospitalar imediata, 9,6\% transferência para serviços especializados, 4,1 \% ficaram em observação no serviço e $1,4 \%$ tiveram internação hospitalar. Vale ressaltar que, nesse mesmo período, 5 casos foram a óbito.

Quanto às notificaçóes acerca dos acidentes e seu local de ocorrência: $47,6 \%$ ocorreram em residência, 3,5\% em escolas, $1,7 \%$ em vias públicas e $0,9 \%$ em creche.

Dos 19 casos que envolveram crianças com deficiência, $42,1 \%$ ocorreram com crianças com deficiência mental, $15,8 \%$ com crianças com deficiência física e $10,5 \%$ com deficiência auditiva.

No que diz respeito ao horário dos acidentes, 27,5\% dos casos ocorreram durante o dia, $13,3 \%$ à noite. Nos demais casos, os horários de ocorrência foram ignorados.

\section{Discussão}

Ao registrar uma maior incidência de acidentes envolvendo crianças do sexo masculino, este estudo confirma uma tendência detectada por outras pesquisas. Para alguns autores, a predominância de acidentes entre meninos pode ser explicada pela maior exposição às atividades dinâmicas que envolvem maior risco, bem como à liberdade adquirida mais precocemente em relação às meninas e à diminuição direta da supervisão de um adulto em suas atividades ${ }^{7,8}$. Assim, a vigilância mais contínua sobre o sexo feminino talvez contribua para uma menor exposiçáo a acidentes ${ }^{8}$.
O desenvolvimento biológico é caracterizado pela aquisição das habilidades motoras grossas e finas, sendo a locomoção a principal habilidade motora grossa adquirida, permitindo que as crianças possam dominar uma variedade maior de atividades. A liberdade irrestrita adquirida por meio da locomoçáo, associada a uma desatenção para os perigos do ambiente, constitui um importante fator para os acidentes na primeira infância? .

Este estudo demonstra a incidência de acidentes entre crianças de 0 a 4 anos (63,9\%). Segundo Filócomo et al. ${ }^{10}$, nesta faixa etária existe uma predominância de ocorrências de acidentes, provavelmente pelo fato da criança ainda não possuir completo domínio de noçôes como distância, velocidade, espaço e tempo; e, quando somando-se a estes fatores, temos a supervisão inadequada do adulto responsável, uma maior exposição ao risco pode ser favorecida ${ }^{10,11}$.

Os dados apontam também para os agentes de intoxicação/envenenamento, em sua maioria produtos químicos e medicamentos, que têm sido relatados como os maiores causadores de agravo na infância. Segundo estudos epidemiológicos, predominam casos de intoxicação em ambiente doméstico, dado que ressalta a importância das campanhas de divulgação para alertar os pais sobre os acidentes tóxicos com crianças e informar a necessidade de um atendimento médico imediato ${ }^{12,13}$.

Quanto aos diagnósticos de lesão mais frequentes (traumatismo superficial múltiplo do pescoço, ferimento da cabeça e traumatismo superficial da cabeça, parte não especificada), pode-se inferir que, pelo fato das quedas serem mais comuns entre crianças, o alto índice de traumas envolvendo a cabeça pode ser resultante desse tipo de acidente $^{14}$.

No que diz respeito à evolução dos casos, a porcentagem de $79,7 \%$ de crianças que tiveram alta hospitalar imediata confirma a literatura, que também aponta para a tendência de recebimento de alta após o tratamento $^{10}$. Uma revisão sistemática da literatura realizada por Martins $^{15}$ indica alta frequência de traumatismos por queda, grande número de atendimentos de baixa complexidade e menor número de óbitos ou casos com encaminhamento para Unidade de Terapia Intensiva ${ }^{10,15}$.

Os estudos que abordam a relação entre deficiência e acidentes são escassos, principalmente no que concerne à literatura nacional. Segundo Wanderer e 
Pedroza ${ }^{16}$, a análise histórica da deficiência nos revela a construção e a consolidação de processos de exclusão social, segregação, preconceito e estigmatização dos indivíduos, caracterizados por diferenças biológicas ou psicológicas tomadas como desvios da normalidade e expressão de menos-valia social. Segundo a literatura, muitos adultos adquirem deficiências como consequência de maus-tratos, abuso sexual na infância ou impedimentos biológicos ocasionados pela violência doméstica contra mulheres durante a gestação $^{16}$. É importante um maior aprofundamento de estudos sobre o tema.

Os dados sobre o local da ocorrência mostraram que $47,6 \%$ dos casos ocorreram em ambiente doméstico e durante o dia, possivelmente no momento em que as crianças desfrutavam de atividades de lazer. Os acidentes domésticos são frequentes, pois nem sempre os pais conhecem as limitaçóes de cada fase da vida dos filhos e, em muitos casos, ignoram os perigos dentro de casa. No Brasil, os acidentes domésticos infantis são causa crescente de morbimortalidade ${ }^{3,17}$.

É importante salientar também os acidentes que ocorreram em escolas e creches, demonstrando a necessidade de que os profissionais de saúde compartilhem sua prática de cuidado com os profissionais do setor da educação e, com uma açáo intersetorial, ampliem conceitos que contribuam com a promoçáo da saúde na redução da morbimortalidade por acidentes ${ }^{18,19}$.

Outro ponto a ser destacado diz respeito à qualidade da informaçáo, ao número de dados ignorados sobre os acidentes na infância, chamando atenção para a importância do preenchimento completo dos registros e da atuação de profissionais de saúde, que podem ser considerados pontos críticos do Sivva, muitas vezes responsáveis pela subnotificação dos dados. $\mathrm{O}$ treinamento das equipes, com o objetivo de reforçar a importância da notificação, também necessita ser incrementado visando à melhoria do sistema ${ }^{20}$.

A redução da incidência de acidentes em crianças pode ser atingida por meio de estudos regionalizados e com o desenvolvimento de programas de prevenção específicos. O eixo principal para a redução dessa incidência deve ser centrado na educaçáo, em um esforço que envolva vários segmentos da população com o intuito de assegurar às crianças e famílias o fornecimento de informaçóes e tratamentos que minimizem o problema ${ }^{21}$.

\section{Conclusão}

As crianças, nos diversos estágios de desenvolvimento, apresentam traumas diferentes, e o conhecimento desse dado é fundamental para a definição de programas eficientes de prevenção. Como demonstrado pelo estudo, o principal tipo de acidente registrado foram as quedas acidentais, ocorridas predominantemente no ambiente doméstico e com crianças do sexo masculino. A partir dessas informaçóes, esta pesquisa buscou demonstrar que estamos diante de crianças vulneráveis, que necessitam de cuidados específicos, principalmente preventivos, em açóes intersetoriais com órgãos e profissionais da saúde e educação envolvidos no acompanhamento do crescimento e desenvolvimento das crianças. A orientação educacional, portanto, demonstra-se essencial para a redução dos acidentes.

\section{Referências}

1. Malta DC, Mascarenhas MDM, Bernal RTI, Viegas APB, Sá NNB, Silva Junior JB. Acidentes e violência na infância: evidências do inquérito sobre atendimentos de emergência por causas externas: Brasil, 2009. Ciênc Saúde Coletiva. 2012;17(9):2247-58.

2. Malta DC, Mascarenhas MDM, Neves ACM, Silva MA. Atendimentos por acidentes e violências na infância em serviços de emergências públicas. Cad Saúde Pública. 2015;31(5):1095-1105.

3. Xavier-Gomes LM, Rocha RM, Andrade-Barbosa TL, Silva CSO. Descrição dos acidentes domésticos ocorridos na infância. Mundo Saúde. 2013;37(4):394-400.

4. Martins CBG. Acidentes e violências na infância e adolescência: fatores de risco e de proteção. Rev Bras Enferm. 2013;66(4):578-84.

5. Gawryszewski VP, Da Silva MMA, Malta DC, Mascarenhas MDM, Costa VC, Matos SG, et al. A proposta da rede de serviços sentinela como estratégia da vigilância de violências e acidentes. Ciênc Saúde Coletiva. 2006;11(Supl):1269-78.

6. São Paulo. Secretaria Municipal de Saúde. Sistema de Informação e Vigilância de Violências e Acidentes - SIVVA [Internet]. São Paulo: Prefeitura de São Paulo; 2013 [citado em 2016 abr 16]. Disponível em: http://www.prefeitura.sp.gov.br

7. Amaral EMS, Silva CLM, Pereira ERR, Guarnieri G, Brito GSS, Oliveira LM. Incidência de acidentes com crianças em um pronto-socorro infantil. Rev Inst Ciênc Saúde. 2009;27(4):313-17.

8. Górios C, Souza RM, Gerolla V, Maso B, Rodrigues CL, Armond JE. Acidentes de transporte de crianças e adolescentes em serviço de emergência de hospital de ensino, Zona Sul da cidade de São Paulo. Rev Bras Ortop. 2014;49(4):391-95.

9. Lima RP, Ximenes LB, Joventino ES, Vieira LJES, Oriá MOB. Principais causas de acidentes domésticos em crianças: um estudo descritivo-exploratório. Online Braz J Nurs. 2008;7(3).

10. 10. Filócomo FRF, Harada MJCS, Silva CV, Pedreira MLG. Estudo dos acidentes na infância em um pronto socorro pediátrico. Rev Latinoam Enf. 2002;10(1):41-7.

11. Regiani C, Correa I. Acidentes na infância em ambiente domiciliar. Rev Min Enf 2006;10(3):277-79. 
12. Ramos CLJ, Targa MBM, Stein AT. Perfil das intoxicaçóes na infância atendidas pelo Centro de Informação Toxicológica do Rio Grande do Sul (CIT/RS), Brasil. Cad Saúde Pública. 2005;21(4):1134-41.

13. Ramos CLJ, Barros HMT, Stein AT, Costa JSD. Fatores de risco que contribuem para o envenenamento pediátrico. J Pediatr. 2010;86(5):435-40.

14. Silveira DC, Pereira JT. Acidentes prevalentes em crianças de 1 a 3 anos em um pronto-socorro de Belo Horizonte no ano de 2007. Rev Min Enferm. 2011;15(2):181-89.

15. Martins CBG. Acidentes na infância e adolescência: uma revisão bibliográfica. Rev Bras Enferm. 2006;59(3):344-48.

16. Wanderer A, Pedroza RLS. A violência como tema transversal aos estudos sobre a deficiência: interconexóes necessárias. Rev Estud Pesq Psicol. 2015;15(1):178-95.

17. Santos BZ, Grosseman S, Silva JYB, Cordeiro MMR, Bosco VL. Injúrias não intencionais na infância: estudo piloto com mães que frequentam a clínica de bebês da Universidade
Federal de Santa Catarina, Brasil. Pesq Bras Odontoped Clin Integr. 2010;10(2):157-61.

18. Vieira LJES, Carneiro RCMM, Frota MA, Gomes ALA, Ximenes LB. Açôes e possibilidades de prevenção de acidentes com crianças em creches de Fortaleza, Ceará. Ciênc Saúde Coletiva. 2009;14(5):1687-97.

19. Rezende MA. Uma proposta de cuidado à criança em creches e pré-escolas: a busca de superação dos determinantes históricos e sociais brasileiros. Acta Paul Enferm. 2004; 17(1):102-7.

20. Souza CS, Costa MCO, Assis SG, Musse JO, Nascimento Sobrinho C, Amaral MTR. Sistema de Vigilância de Violências e Acidentes/VIVA e a notificação da violência infanto-juvenil, no Sistema Único de Saúde/SUS de Feira de Santana-Bahia, Brasil. Ciênc Saúde Coletiva. 2014;19(3):773-84.

21. Brito MA, Rocha SS. A criança vítima de acidentes domésticos sob o olhar das teorias de enfermagem. J Res Fund Care Online. 2015;7(4):3351-65.

\section{Como citar este artigo:}

Rodrigues CL, Armond JE, Iamarino APM, Santos ACG, Armond RE, Górios C. Notificaçôes de acidentes pediátricos em São Paulo. Rev. Aten. Saúde. 2017;15(51)52-56. 